\title{
Evolution of serum biochemical indicators in anorexia nervosa patients: a 1-year follow-up study
}

\author{
E. Nova, ${ }^{*}$ I. Lopez-Vidriero, $\uparrow$ P. Varela,$\ddagger$ J. Casas $\S \&$ A. Marcos* \\ *Departamento de Metabolismo y Nutrición, Instituto del Frío, CSIC; †Centro Nacional de Biotecnología, Unidad de Genómica, \\ CSIC; †Departamento de Nutrición y Bromatología I, Universidad Complutense de Madrid; §Servicio de Pediatría, Hospital de \\ Móstoles, Madrid, Spain
}

\section{Correspondence}

Esther Nova $\mathrm{PhD}$,

Departamento de Metabolismo y

Nutrición. Instituto del Frío,

CSIC,

C/Jose Antonio Novais 10,

28040 Madrid,

Spain.

Tel.: +34915490038

Fax: +34 915493627

E-mail: enova@if.csic.es

\section{Keywords}

follow-up

study, red blood cells, refeeding, restricting-type anorexia nervosa, serum biochemical markers, serum enzymatic activities.

\begin{abstract}
Background Long-term studies on the evolution of serum biochemical indicators in anorexia nervosa (AN) patients during treatment are lacking in the literature. Thus, a 1-year follow-up of serum biochemical parameters in a homogeneous group of AN patients was performed.
\end{abstract}

Methods Fourteen restricting-type AN patients were studied on admission to hospital, after 1 month of inpatient treatment and after 6 and 12 months after admission.

Results Red blood cell count (RBC) and haemoglobin, serum glucose, total protein and the enzyme activities aspartate aminotransferase (AST), alkaline phosphatase (AlP), lactate dehydrogenase $(\mathrm{LDH})$ and creatine kinase (CK) were significantly lower in patients on admission than in the control group. Total protein, high-density lipoprotein cholesterol (HDL-c), AST, AlP and CK showed significant changes among time points (ANOVA, $P<0.05$ ). Significant correlations were found between the change in RBC, haemoglobin, haematocrit, and the change in weight and body mass index $(r=0.74-0.86 ; P<0.01)$. High cholesterol and amylase activity were found at all time points. While AST, LDH and CK reached control values within 6 months of treatment, AlP was always lower.

Conclusion Serum AlP, hypercholesterolaemia and RBC seem to need longer periods of treatment with further weight gain to fully normalize. Therefore, these parameters should be monitored in AN patients long-term follow-up. 


\section{Conflict of interests, source of funding and authorship}

The authors have no conflicts of interest to disclose. No source of finding needs to be acknowledged regarding this work.

AM, PV and JC discussed core ideas of protocol design; EN and ILV were responsible for data collection; JC facilitated patients' recruitment; EN, PV, ILV and AM performed the interpretation of data; EN and AM wrote the manuscript.

\section{Introduction}

The malnourished condition of patients with anorexia nervosa (AN) affects all organs and systems in the body. The extent of the metabolic changes depends on the duration and severity of the reduced food intake and the amount of weight lost. Serum biochemical markers of nutritional status and metabolism are routinely examined in AN patients after diagnosis; however, most markers are usually normal. This has led to the conclusion that adaptive mechanisms preserve their nutritional status until advanced stages of debilitation (Lucas, 1977). This might be the reason why only a few papers have been published containing values for these routine biochemical parameters in AN patients. However, some of these studies have been carried out in groups that showed poor homogeneity and included subjects who could have belonged to different subtypes or even to different categories of eating disorders (Pryor et al., 1996). This is of importance since studies in AN patients have shown that the nutritional status in these patients varies according to factors such as the duration of the illness prior to treatment or the different types of compensatory behaviours adopted to control weight (Marcos, 1997). Moreover, the studies published are mainly of two types; cross-sectional studies (Pertschuk et al., 1982; Dowd et al., 1983; Mira et al., 1987, 1989; Van Binsbergen et al., 1988; Varela et al., 1992) or studies comparing a group of AN patients before and after nutritional rehabilitation (Wyatt et al., 1982; Barbe et al., 1993; Megia et al., 1994; Fink et al., 1996; Smith et al., 1996). There are no studies in the literature measuring biochemical markers in AN patients in long-term follow-up.

On this basis, the authors have evaluated some serum biochemical parameters in a follow-up study of a homogenous group of AN patients diagnosed with the restricting-type of the illness, and receiving the same multidisciplinary treatment. The aim of the study was to measure these parameters at different time points during inhospital and outpatient periods of treatment in a 1-year follow-up.

\section{Methods}

\section{Subjects and study design}

A group of 14 adolescent AN patients recruited on admission to the Hospital de Móstoles (Madrid, Spain) was included in this 1-year followup study. All the patients met criteria for the diagnosis of AN. To increase the homogeneity of the study group only female patients in the adolescent age (12-18 years), undergoing their first hospital admission and with restricting type AN (DSM IV) (American Psychiatric Association, 1994) were included. The follow-up covered inpatient and outpatient periods of treatment for the 14 patients, and thus, four different time points were considered: (i) when AN patients had been recently admitted to a rehabilitation programme in the hospital $\left(t_{0}\right)$; (ii) 1 month later $\left(t_{1}\right)$; (iii) 6 months after admission $\left(t_{6}\right)$; and (iv) 12 months after admission $\left(t_{12}\right)$. In all, 26 patients were first recruited for the study but 12 were considered drop outs after failing to comply with any of the dates (time points) in the study. The results obtained are limited to those 14 patients. At $t_{0}$, the mean duration of the illness was relatively short ( $9.4 \pm 6.3$ months). No differences were observed in age, weight, or duration of the illness at admission between those patients who did not finish the study and those who did. At $t_{0}$ and $t_{1}$, all patients were in hospital, where as at $t_{6}$ and $t_{12}$, there were 12 and 13 outpatients and two and one inpatients respectively. In the course of the year, six out of the 14 patients were readmitted; four of them on one occasion and two patients had up to two 
more admissions in the year. Fifteen healthy subjects matched by age, sex and sociocultural background were recruited from a school and were used as a control group. All subjects gave their full and informed consent once the purpose and nature of the study had been explained. Parental consent was also obtained for those under 18 years of age. The study was conducted in accordance with the ethical rules of the Helsinki Declaration as revised in 1983 and the EEC Good Clinical Practice guidelines (document 111/ $3976 / 88$ of July 1990) and the study protocol was approved by the Ethics Committee of the Hospital de Mostoles.

\section{Treatment protocol}

According to the Hospital of Mostoles' refeeding program for AN patients, energy intake varied between 2090 and $4180 \mathrm{~kJ} \mathrm{day}^{-1}(500-1000 \mathrm{kcal}$ $\mathrm{day}^{-1}$ ) for the first 2-4 days of the hospital admission, and was increased by $2090 \mathrm{~kJ}$ (500 kcal) every 3-4 days to ensure an acceptable weight gain of $0.5-1.5 \mathrm{~kg} \mathrm{week}^{-1}$ at the beginning of the treatment and 200-400 $\mathrm{g} \mathrm{week}^{-1}$ thereafter. During the weight gain period, four solid meals plus two liquid supplements, if necessary, were offered to the patient. Dietary management was supported by psychological therapy to correct eating behaviour. As inpatients, some patients were allowed to do rehabilitation exercises in a fitness room for half an hour a day. On admission, an eventual discharge weight was established by the treating physician, which was within one SD (standard deviation) of the ideal body weight (IBW) for age and height. When patients entered outpatient treatment, a normocaloric diet was prescribed together with outpatient psychological therapy.

\section{Measures and biochemical analyses}

At every time point of the study, basic anthropometric data were obtained by the standard procedure after an overnight fast. Weight and height were determined without clothes and shoes, using a digital electronic weighing scale (Seca alpha; Igmy, France; range: $0.1-150 \mathrm{~kg}$ ) and a digital stadiometer (Harpender Pfifter 450; Carlstadt, NJ, USA; range 0.70-2.05 m) respectively. From these data, body mass index (BMI) [weight $(\mathrm{kg}) /$ height $^{2}$ $\left.\left(\mathrm{m}^{2}\right)\right], Z$-score of the BMI and IBW percentage (IBW $\%)$ according to Spanish growth reference charts (Hernández et al., 1988) were calculated. Blood was also collected in the early morning by puncture of the cubital vein under fasting conditions. Two millilitre was collected in EDTA- $\mathrm{K}_{3}$ tubes for haematology analysis and 6-7 mL in gel-containing tubes for serum separation. Red blood cell counts (RBC), haemoglobin, haematocrit and corpuscular indexes were analysed in an automatic cell counter (Technicon-Bayer H1). The following biochemical parameters were assessed in serum samples using a Merck autoanalyser AU 510 (Merck, Hamburg, Germany): glucose, total protein, urea, uric acid, creatinine, total bilirrubin, lipid profile (total cholesterol and lipoprotein-cholesterol fractions) and the enzyme activities of aspartate aminotransferase (AST), alanine aminotransferase (ALT), gamma-glutamil transferase $(\gamma-\mathrm{GT})$, alkaline phosphatase (AlP), lactate dehydrogenase (LDH), creatine kinase (CK) and amylase.

\section{Statistical analysis}

All values reported are mean \pm SD. Data were analysed by repeated-measures ANOVA. When the analysis indicated a significant effect of time $(P<0.05)$, the Bonferroni method was used for a pairwise comparison between the four time points and for calculation of $95 \%$ confidence limits for the differences between the time points. Values of $P<0.05$ were considered significant. The Bonferroni method encompasses a downward adjustment of significance limits for the differences between the time points. All $P$-values were two-tailed. Differences between the AN group and the control group were assessed by Student's $t$-test. Differences were considered statistically significant at $P<0.05$. The variables' change between time points was also calculated and the associations between the biochemical and anthropometrical parameters' changes in time intervals were assessed using Pearson's correlation test. All the tests were performed by the SPSS 11.0.1 (SPSS Inc., Chicago, IL, USA). 
Table 1 Anthropometrical parameters in controls and patients with anorexia nervosa at different time points during a 1-year follow-up

\begin{tabular}{|c|c|c|c|c|c|c|}
\hline & Controls & $t_{0}$ & $t_{1}$ & $t_{6}$ & $t_{12}$ & $\begin{array}{l}\text { Repeated-measures } \\
\text { ANova } P \text {-value }\end{array}$ \\
\hline Age (years) & $15.1 \pm 1.4$ & $15.1 \pm 2.6$ & $15.2 \pm 2.6$ & $15.6 \pm 2.6$ & $16.1 \pm 2.6$ & $<0.001$ \\
\hline Height $(\mathrm{cm})$ & $161.7 \pm 5.4$ & $160.6 \pm 6.3$ & $160.8 \pm 6.1$ & $160.9 \pm 6.3$ & $161.2 \pm 6.5$ & 0.206 \\
\hline Weight (kg) & $53.0 \pm 5.0$ & $39.4 \pm 6.1^{* * a, b, c}$ & $43.0 \pm 4.9^{* *, a}$ & $44.8 \pm 4.5^{* *, b}$ & $45.2 \pm 6.7^{* *, c}$ & $<0.001$ \\
\hline BMI $\left(\mathrm{kg} \mathrm{m}^{-2}\right)$ & $20.2 \pm 1.3$ & $15.2 \pm 1.7^{* * a, b, c}$ & $16.6 \pm 1.1^{* *, a}$ & $17.3 \pm 1.1^{* *, \mathrm{~b}}$ & $17.3 \pm 2.0^{* *, c}$ & $<0.001$ \\
\hline Score Z (BMI) & $-0.12 \pm 0.48$ & $-1.93 \pm 0.78^{* *, b, c}$ & $-1.44 \pm 0.53 * *, b$ & $-1.29 \pm 0.61 * *, c$ & $-1.33 \pm 0.96 *$ & 0.002 \\
\hline IBW (\%) & $94.6 \pm 7.6$ & $72.2 \pm 7.9^{* *, a, c, d}$ & $78.7 \pm 6.0^{* *, a}$ & $82.1 \pm 7.2^{* *, c}$ & $82.5 \pm 10.0^{*, d}$ & $<0.001$ \\
\hline Weight gain (kg) & - & - & $3.6 \pm 2.1$ & $5.4 \pm 3.7$ & $5.8 \pm 4.5$ & 0.179 \\
\hline
\end{tabular}

BMI, body mass index; IBW, ideal body weight.

Values are given as mean \pm SD.

Significant differences against Controls (Student's $t$-test, independent data): ${ }^{*} P \leq 0.01 ;{ }^{*} P \leq 0.001$.

Significant differences between mean values with common superscripts (Bonferroni test): ${ }^{\mathrm{a}, \mathrm{b}} P \leq 0.01 ;{ }^{\mathrm{c}, \mathrm{d}} P \leq 0.001$.

\section{Results}

Anthropometrical parameters are presented in Table 1. While height was similar in patients and controls, weight and BMI were significantly lower in all the stages in the AN group. Nevertheless, a significant increase was observed in weight and BMI at $t_{1}$, both parameters remaining unmodified thereafter. RBC and related parameters are shown in Table 2. No significant changes were observed in the red blood cells by repeated-measures ANOVA, except for mean corpuscular haemoglobin $(\mathrm{MCH})$, which showed a significant increase in $t_{12}$ compared to $t_{6}$. Eleven out of 14 patients showed $\mathrm{MCH}$ values above the normal range in $t_{12}$. Significantly lower values compared to the control group were found for RBC, haemoglobin and MCH concentration $(\mathrm{MCHC})$ in $t_{0}$, while significantly higher values were observed for mean corpuscular vol- ume. A nonsignificant trend was observed towards increasing values of $\mathrm{RBC}$ and haemoglobin during the 1-year follow-up. In addition, the changes in RBC, haemoglobin and haematocrit between $t_{0}$ and $t_{12}$ were positively correlated with the changes in weight, IBW (\%) and BMI in this time interval ( $r$ between 0.74 and $0.86 ; P<0.01$ ).

Serum biochemical parameters are shown in Table 3. Changes during the follow-up were observed for total protein, HDL-c and the enzymes AST, AlP and CK. Total protein, HDL-c and CK increased significantly at $t_{6}$ and $t_{12}$ compared to the inpatient period of treatment. AST also increased along the follow-up reaching at $t_{12}$ significantly higher values than at $t_{0}$ and $t_{1}$, and AlP increased significantly already during the inpatient treatment $\left(t_{1}\right.$ versus $\left.t_{0}\right)$ and was maintained unmodified thereafter. All these parameters showed lower values than the control group on

Table 2 Red blood cells (RBC) and haematological indices in controls and anorexia nervosa patients at different time points during a 1-year follow-up

\begin{tabular}{|c|c|c|c|c|c|c|}
\hline & Controls & $t_{0}$ & $t_{1}$ & $t_{6}$ & $t_{12}$ & $\begin{array}{l}\text { Repeated-measures } \\
\text { ANOVA } P \text {-value }\end{array}$ \\
\hline $\operatorname{RBC}\left(\times 10^{12} L^{-1}\right)$ & $4.9 \pm 0.3$ & $4.2 \pm 0.4^{* * *}$ & $4.0 \pm 0.3 * * *$ & $4.3 \pm 0.3 * * *$ & $4.3 \pm 0.3 * * *$ & 0.523 \\
\hline Haemoglobin $\left(\mathrm{g} \mathrm{dL}^{-1}\right)$ & $14.8 \pm 1.2$ & $12.9 \pm 1.5^{* *}$ & $12.8 \pm 1.0^{* * *}$ & $13.2 \pm 0.8^{* * *}$ & $13.7 \pm 1.2^{*}$ & 0.327 \\
\hline Haematocrit (\%) & $40.7 \pm 3.3$ & $38.6 \pm 4.2$ & $38.0 \pm 3.3^{*}$ & $39.8 \pm 3.0$ & $40.6 \pm 3.1$ & 0.444 \\
\hline MCV (fl) & $83.0 \pm 3.8$ & $92.9 \pm 3.7^{* * *}$ & $94.6 \pm 38^{* * *}$ & $93.6 \pm 3.9 * * *$ & $94.1 \pm 2.9 * * *$ & 0.185 \\
\hline $\mathrm{MCH}(\mathrm{pg})$ & $30.1 \pm 1.8$ & $30.9 \pm 1.4$ & $32.0 \pm 1.9 *$ & $30.9 \pm 1.4^{\mathrm{a}}$ & $31.8 \pm 1.3^{* *, a}$ & $<0.05$ \\
\hline $\mathrm{MCHC}\left(\mathrm{g} \mathrm{dL}^{-1}\right)$ & $36.3 \pm 1.2$ & $33.6 \pm 1.4^{* * *}$ & $33.8 \pm 1.53^{* * *}$ & $33.1 \pm 1.0^{* * *}$ & $33.8 \pm 0.8 * * *$ & 0.165 \\
\hline
\end{tabular}

$\mathrm{MCV}$, mean corpuscular volume; $\mathrm{MCH}$, mean corpuscular haemoglobin; $\mathrm{MCHC}$, mean corpuscular haemoglobin concentration. Values are given as mean \pm SD.

Significant differences against controls (Student's $t$-test, independent data): ${ }^{*} P \leq 0.05 ;{ }^{* *} P \leq 0.01 ;{ }^{* *} P \leq 0.001$.

Significant differences between mean values with common superscripts (Bonferroni test): ${ }^{a} P \leq 0.05$. 
Table 3 Serum biochemical parameters in controls and anorexia nervosa patients at different time points during a 1-year followup

\begin{tabular}{|c|c|c|c|c|c|c|}
\hline & Controls & $t_{0}$ & $t_{1}$ & $t_{6}$ & $t_{12}$ & $\begin{array}{l}\text { Repeated-measures } \\
\text { ANOVA } P \text {-value }\end{array}$ \\
\hline $\begin{array}{l}\text { Glucose } \\
\left(\mathrm{mg} \mathrm{dL}^{-1}\right)\end{array}$ & $86.6 \pm 4.5$ & $77.4 \pm 7.7^{* * *}$ & $78.8 \pm 17.2$ & $83.8 \pm 12.7$ & $86.4 \pm 8.4$ & 0.252 \\
\hline $\begin{array}{l}\text { Total protein } \\
\left(\mathrm{g} \mathrm{dL}^{-1}\right)\end{array}$ & $7.47 \pm 0.27$ & $6.98 \pm 0.67^{*, a, b}$ & $6.96 \pm 0.91$ & $7.70 \pm 0.52^{a}$ & $7.47 \pm 0.30^{b}$ & $<0.01$ \\
\hline $\begin{array}{l}\text { Urea } \\
\qquad\left(\mathrm{mg} \mathrm{dL}{ }^{-1}\right)\end{array}$ & $23.8 \pm 5.0$ & $39.8 \pm 10.7^{* * *}$ & $37.9 \pm 10.8^{* * *}$ & $38.8 \pm 9.7^{* * *}$ & $38.6 \pm 10.5^{* * *}$ & 0.918 \\
\hline $\begin{array}{l}\text { Uric acid } \\
\qquad\left(\mathrm{mg} \mathrm{dL}^{-1}\right)\end{array}$ & $4.2 \pm 0.6$ & $3.7 \pm 0.9$ & $3.7 \pm 0.7^{*}$ & $3.8 \pm 0.8$ & $4.2 \pm 1.1$ & 0.578 \\
\hline $\begin{array}{l}\text { Creatinine } \\
\left(\mathrm{mg} \mathrm{dL}^{-1}\right)\end{array}$ & $0.87 \pm 0.09$ & $0.89 \pm 0.12$ & $0.88 \pm 0.13$ & $0.99 \pm 0.17^{*}$ & $0.96 \pm 0.11^{*}$ & 0.111 \\
\hline $\begin{array}{l}\text { Total bilirubin } \\
\left(\mathrm{mg} \mathrm{dL}^{-1}\right)\end{array}$ & $0.38 \pm 0.26$ & $0.38 \pm 0.23$ & $0.34 \pm 0.22$ & $0.35 \pm 0.18$ & $0.37 \pm 0.17$ & 0.835 \\
\hline $\mathrm{TG}\left(\mathrm{mg} \mathrm{dL}^{-1}\right)$ & $71.5 \pm 33.1$ & $69.4 \pm 30.2$ & $58.7 \pm 31.2$ & $64.6 \pm 24.0$ & $64.7 \pm 20.3$ & 0.185 \\
\hline $\begin{array}{l}\mathrm{CHOL} \\
\left(\mathrm{mg} \mathrm{dL}^{-1}\right)\end{array}$ & $163.9 \pm 27.8$ & $208.2 \pm 61.8^{*}$ & $208.0 \pm 49.2^{* *}$ & $225.7 \pm 63.6^{* *}$ & $208.4 \pm 41.2^{* *}$ & 0.363 \\
\hline $\begin{array}{l}\mathrm{HDL}-\mathrm{C} \\
\left(\mathrm{mg} \mathrm{dL}^{-1}\right)\end{array}$ & $59.5 \pm 7.3$ & $45.1 \pm 18.3^{* *, \mathrm{c}}$ & $46.9 \pm 11.9^{* *, \mathrm{~d}, \mathrm{e}}$ & $52.6 \pm 13.4^{d}$ & $61.6 \pm 18.0^{\mathrm{c}, \mathrm{e}}$ & $<0.01$ \\
\hline $\begin{array}{l}\text { LDL-C } \\
\left(\mathrm{mg} \mathrm{dL}^{-1}\right)\end{array}$ & $90.0 \pm 23.7$ & $154.5 \pm 65.2$ & $149.4 \pm 50.6^{* * *}$ & $162.4 \pm 67.6$ & $133.8 \pm 54.6^{* *}$ & 0.455 \\
\hline $\begin{array}{l}\text { VLDL-c } \\
\left(\mathrm{mg} \mathrm{dL}^{-1}\right)\end{array}$ & $14.3 \pm 6.6$ & $13.9 \pm 6.0$ & $11.7 \pm 6.2$ & $12.9 \pm 4.8$ & $12.9 \pm 4.1$ & 0.185 \\
\hline AST $\left(U L^{-1}\right)$ & $16.0 \pm 2.2$ & $11.4 \pm 4.4^{* *, f}$ & $12.4 \pm 2.6^{* * * . g}$ & $15.2 \pm 3.6$ & $17.9 \pm 5.1^{f, g}$ & $<0.01$ \\
\hline ALT $\left(U L^{-1}\right)$ & $11.3 \pm 2.3$ & $10.6 \pm 4.4$ & $10.1 \pm 3.2$ & $10.3 \pm 2.9$ & $12.6 \pm 5.4$ & 0.578 \\
\hline$\gamma-G T\left(U L^{-1}\right)$ & $10.0 \pm 2.0$ & $11.4 \pm 4.1$ & $11.9 \pm 3.9$ & $13.1 \pm 6.6$ & $13.0 \pm 5.0$ & 0.846 \\
\hline AIP $\left(U L^{-1}\right)$ & $325.0 \pm 164.8$ & $119.4 \pm 37.4^{* * *, h, i, j}$ & $164.5 \pm 68.7^{* *, h}$ & $190.3 \pm 97.6^{*, i}$ & $192.1 \pm 117.1^{*, j}$ & $<0.01$ \\
\hline $\mathrm{LDH}\left(\mathrm{U} \mathrm{L}^{-1}\right)$ & $312.8 \pm 53.4$ & $248.9 \pm 84.1^{*}$ & $269.0 \pm 45.1^{*}$ & $281.6 \pm 62.9$ & $267.5 \pm 66.6$ & 0.462 \\
\hline$C K\left(U L^{-1}\right)$ & $60.7 \pm 21.6$ & $31.7 \pm 14.5^{* * *, k, l}$ & $36.3 \pm 10.2^{* *, m, n}$ & $65.2 \pm 32.8^{\mathrm{k}, \mathrm{m}}$ & $71.7 \pm 27.3^{1, \mathrm{n}}$ & 0.001 \\
\hline $\begin{array}{l}\text { Amylase } \\
\left(U L^{-1}\right)\end{array}$ & $58.0 \pm 12.8$ & $74.1 \pm 18.0^{* *}$ & $101.6 \pm 57.6^{*}$ & $75.2 \pm 50.9$ & $75.1 \pm 21.0^{*}$ & 0.249 \\
\hline
\end{tabular}

TG, triglycerides; CHOL, total cholesterol; HDL-c, high-density lipoprotein cholesterol; LDL-c, low-density lipoprotein cholesterol; VLDL-c, verylow-density lipoprotein cholesterol; AST, aspartate aminotransferase; ALT, alanine aminotransferase; $\gamma$-GT, gamma-glutamil transferase; AIP, alkaline phosphatase; LDH, lactate dehydrogenase; CK, creatine kinase. $U, 1$ unit is that quantity of an enzyme which transforms $1 \mu$ mol of substrate per minute under standardized conditions.

Values are given as mean \pm SD.

Significant differences against controls (Student's $t$-test, independent data): ${ }^{*} P \leq 0.05 ;{ }^{*} P \leq 0.01 ;{ }^{*} * P \leq 0.001$

Significant differences between mean values with common superscripts (Bonferroni test): ${ }^{c, d, e, f, g, i, j, m} P \leq 0.05 ;{ }^{a, b, h, k, n} P \leq 0.01 ;{ }^{l} P \leq 0.001$.

admission but reached control values at $t_{6}$ and $t_{12}$ except for AlP that was maintained below control values throughout the study. Glucose and LDH also showed values lower than the control group at $t_{0}$ but reached control values with refeeding. Urea, total cholesterol, low-density lipoprotein cholesterol (LDL-c) and amylase concentrations in serum were higher than in the control group on admission and at all subsequent time points.

A significant correlation was observed between AlP activity and the $Z$-score of the BMI at $t_{0}, t_{1}$ and $t_{6} \quad(r=0.80, P<0.001 ; r=0.71, P<0.01$ and $r=0.61, P<0.05$ respectively). In addition, a negative correlation was found between the change in AlP activity from $t_{0}$ to $t_{1}$ and the increase in weight during the same period $(r=-0.86 ; P<0,001)$.

\section{Discussion}

The results found in this group of restricting-type adolescent AN patients showed that serum biochemical parameters measured on hospital admission are impaired in comparison with the control group. Protein, glucose and most enzyme activities are low in response to the restricted nutrient intake, and return to normal either during admission or during the outpatient period of treatment. However, other parameters such as $\mathrm{RBC}, \mathrm{AlP}$, cholesterol and amylase, which did not 
normalize during the 1-year follow-up, appear to be linked to the impaired weight status of the patients which was still observed after 1-year of treatment. Mean weight and BMI on admission were under the third percentile according to the Spanish growth reference charts (Hernández et al., 1988), and did not reach the 10th percentile after the 1-year period of treatment.

The low values found for $\mathrm{RBC}$, haemoglobin and $\mathrm{MCHC}$ on admission are in agreement with results published by other authors in AN patients before treatment (Palla \& Litt, 1988; Devuyst et al., 1993). Six out of the 14 patients had values below the normal range for $\mathrm{RBC}$, haematocrit and $\mathrm{MCHC}$ and four of them had haemoglobin below the normal range. All cases of anaemia found in our group were normocytic, except one case of iron deficiency anaemia. Iron deficiencies are infrequent because the amenorreic status of anorexic patients decreases iron requirements. The increase in the cells' volume and in the $\mathrm{MCH}$ seems to be an adaptive mechanism to the nutrient deficiency, thus, trying to compensate for moderately reduced $\mathrm{RBC}$ and haemoglobin concentrations.

No significant improvement was found in RBC and haemoglobin with refeeding, and they remained lower than in the control group at all time points. Moreover, the correlations found between the change in red cells and haemoglobin and the change in anthropometrical parameters reflect that their improvement seems to be secondary to the nutritional status recovery and thus, dependent on the availability of substrates for anabolic processes. Increased metabolic demands for iron and zinc during refeeding and nutritional recovery have been referred previously in this group of patients (Nova et al., 2004).

Regarding serum biochemistry, reduced glucose and protein concentrations as well as low AST and LDH activities compared to those of the healthy girls reflect energy and protein sparing strategies in response to the reduced food intakes. Glucose and protein were recovered early once refeeding begins, i.e. in the first month, while enzyme activities normalized in the first 6 months. In addition, the low AlP values might reflect a reduction in the anabolic processes occurring normally at this age of rapid growth, while low CK activity could be a consequence of the probably reduced muscle mass. A previous study has described low serum AlP activity in AN patients that was not due to a deficiency in zinc, the enzyme cofactor, as plasma and hair zinc levels were normal in these patients (Varela et al., 1992). In the current study, five subjects in the control group showed AlP values above the normal range. Under these circumstances, the increase of AlP in $t_{1}$ in the AN patients indicates an improvement in their nutritional status. In addition, the negative correlation found between the change in AlP and the change in weight between $t_{0}$ and $t_{1}$ shows that the increase in AlP observed in $t_{1}$ occurred in those patients whose weight is less depleted on admission and therefore gain less weight during the first month of treatment. Similar results were found by Heer et al. (2004) who also found a significant increase in bone AlP with nutritional therapy in AN patients.

Other authors have found relatively frequent increased values of aminotransferases (Palla \& Litt, 1988; Van Binsbergen et al., 1988). This can be due to a number of reasons such as fatty liver, hepatic failure, dehydration or antidepressant therapy. However, the patients in this study always showed AST and ALT values within the normal range. The high urea values in serum are attributable to the relatively high protein intakes that are commonly found in these patients. In fact, in our patients, the relative protein intake (\% of total energy) was higher than in controls in all the stages (Nova et al., 2001). A simultaneous increase in urea and creatinine values suggests increased muscle catabolism, but this situation was only found in one patient in $t_{6}$, who was experiencing acute weight loss and was severely underweight. According to the literature and the results in this study, hypercholesterolaemia is present in approximately $50 \%$ of the AN patients (Mordasini et al., 1978; Mira et al., 1987; Arden et al., 1990). LDL-c increase is mostly determined by the severe loss of body fat and the resulting changes in thyroid hormones, increased lipolysis and decreased endogenous cholesterol synthesis accompanied by decreased LDL removal (Weinbrenner et al., 2004). However, although refeeding did not decrease LDL-c, it decreased the percentage of 
patients showing high cardiovascular risk indexes (triglycerides/HDL-c and LDL-c/HDL-c). Finally, although high serum amylase values are typical of patients with purging behaviour and salivary gland enlargement, previous studies (Scheutzel \& Gerlach, 1991; Kinzl et al., 1993) have described high serum $\alpha$-amylase in approximately $20 \%$ of restricting AN patients. A study of the changes in salivary amylase in serum and parotid gland in rats have shown that feeding in fasted state results in a decrease in parotid tissue amylase activity and a proportional increase in serum levels of parotid isoamylase (Nagy et al., 2001).

In conclusion, this study showed that serum biochemical parameters reflect the compromised nutritional status of the restricting AN patients on admission to hospital. Enzymatic activities, except AlP, reach control values during the outpatient period of treatment, despite total anthropometrical recovery not being achieved at the end of the follow-up. Serum AlP activity, hypercholesterolaemia and red cells seem to need more prolonged periods of treatment with further weight gain to fully normalize. Therefore, these are parameters worth monitoring during AN patients long-term follow-up, as they might reflect good or poor response to treatment for individual patients.

\section{References}

American Psychiatric Association (1994) Diagnostic and Statistical Manual of Mental Disorders, 4th edn. Washington, DC: American Psychiatric Association.

Arden, M.R., Weiselberg, E.C., Nussbaum, M.P., Shenker, R. \& Jacobson, M.S. (1990) Effect of weight restoration on the dyslipoproteinemia of anorexia nervosa. J. Adolesc. Health Care 11, 199-202.

Barbe, P., Bennet, A., Stebenet, M., Perret, B. \& Louvet, J.P. (1993) Sex-hormone-binding globulin and protein-energy malnutrition indexes as indicators of nutritional status in women with anorexia nervosa. Am. J. Clin. Nutr. 57, 319-322.

Devuyst, O., Lambert, M., Rodhain, J., Lefebvre, C. \& Coche, E. (1993) Haematological changes and infectious complications in anorexia nervosa: a case control study. Q. J. Med. 86, 791-799.

Dowd, P.S., Kelleher, J., Walker, B.E. \& Guillon, P.J. (1983) Nutritional and immunological assessment of patients with anorexia nervosa. Clin. Nutr. 2, 79-83.

Fink, S., Eckert, E., Mitchell, J., Crosby, R. \& Pomeroy, C. (1996) T-lymphocyte subsets in patients with abnormal body weight: longitudinal studies in anorexia nervosa and obesity. Int. J. Eat. Disord. 20, 295-305.

Heer, M., Mika, C., Grzella, I., Heussen, N. \& HerpertzDahlmann, B. (2004) Bone turnover during inpatient nutritional therapy and outpatient follow-up in patients with anorexia nervosa compared with that in healthy control subjects. Am. J. Clin. Nutr. 80, 774-781.

Hernández, M., Castellet, J., Narvaiza, J.L., Rincón, J.M., Ruiz, E., Sánchez, E., Sobradillo, B. \& Zurimendi, A. (1988) Curvas y Tablas de Crecimiento (Growth tables and charts). Instituto de Investigación sobre Crecimiento $y$ Desarrollo. Madrid: Fundación Faustino Orbegozo, Carsi.

Kinzl, J., Biebl, W. \& Herold, M. (1993) Significance of vomiting for hyperamylasemia and sialadenosis in patients with eating disorders. Int. J. Eat. Disord. 13, 117-124.

Lucas, A. (1977) On the meaning of laboratory values in anorexia nervosa. Mayo Clin. Proc. 52, 748-750.

Marcos, A. (1997) The immune system in eating disorders: an overview. Nutrition 13, 853-862.

Megia, A., Gil-Canalda, I., Luna, R., Herranz, L., Weisz, P., Bacaicoia, A., Cos, A. \& Gómez-Candela, C. (1994) Our experience in the nutritional treatment of anorexia nervosa (1989-1991). Nutr. Hosp. 9, 400-406.

Mira, M., Stewart, P.M., Vizzard, J. \& Abraham, S. (1987) Biochemical abnormalities in anorexia nervosa and bulimia. Ann. Clin. Biochem. 24, 29-35.

Mira, M., Stewart, P.M. \& Abraham, S.F. (1989) Vitamin and trace element status of women with disordered eating. Am. J. Clin. Nutr. 50, 940-944.

Mordasini, R., Klose, G. \& Greten, H. (1978) Secondary type II hyperlipoproteinemia in patients with anorexia nervosa. Metabolism 27, 71-79.

Nagy, A., Barta, A., Varga, G. \& Zelles, T. (2001) Changes of salivary amylase in serum and parotid gland during pharmacological and physiological stimulation. J. Physiol. Paris 95, 141-145.

Nova, E., Varela, P., López-Vidriero, I., Toro, O., Casas, J., Ceñal, M.J. \& Marcos, A. (2001) A one-year follow-up study in anorexia nervosa. Dietary pattern and anthropometrical evolution. Eur. J. Clin. Nutr. 55, 547-554.

Nova, E., Lopez-Vidriero, I., Varela, P., Toro, O., Casas, J. \& Marcos, A. (2004) Indicators of nutritional status in restricting-type anorexia nervosa patients. A one-year follow-up study. Clin. Nutr. 23, 1353-1359.

Palla, B. \& Litt, I.F. (1988) Medical complications of eating disorders in adolescents. Pediatrics 81, 613-623.

Pertschuk, M.J., Crosby, L.O., Barot, L. \& Mullen, J.L. (1982) Immunocompetency in anorexia nervosa. Am. J. Clin. Nutr. 35, 968-972.

Pryor, T., Wiederman, M.W. \& McGilley, B. (1996) Clinical correlates of anorexia nervosa subtypes. Int. J. Eat. Disord. 19, 371-379. 
Scheutzel, P. \& Gerlach, U. (1991) Alpha-amylase isoenzymes in serum and saliva of patients with anorexia and bulimia nervosa. Z. Gastroenterol. 29, 339-345.

Smith, G., Robinson, P.H. \& Fleck, A. (1996) Serum albumin distribution in early treated anorexia nervosa. Nutrition 12, 677-684.

Van Binsbergen, C.J.M., Okink, J., Van Den Berg, H., Koppeschaar, H. \& Colelingh Bennink, H.J.T. (1988) Nutritinal status in anorexia nervosa: clinical chemistry, vitamins, iron and zinc. Eur. J. Clin. Nutr. 42, 929-937.

Varela, P., Marcos, A. \& Navarro, P. (1992) Zinc status in anorexia nervosa. Ann. Nutr. Metab. 36, 197-202.
Weinbrenner, T., Zuger, M., Jacoby, G.E., Herpertz, S., Liedtke, R., Sudhop, T., Gouni-Berthold, I., Axelson, M. \& Berthold, H.K. (2004) Lipoprotein metabolism in patients with anorexia nervosa: a case-control study investigating the mechanisms leading to hypercholesterolaemia. Br. J. Nutr. 91, 959-969.

Wyatt, R.J., Farrell, M., Berry, P.L., Forristal, J., Maloney, M.J. \& West, C. (1982) Reduced alternative complement pathway control protein levels in anorexia nervosa: response to parenteral alimentation. Am. J. Clin. Nutr. 35, 973-980. 